\title{
A UHF-RFID gate control system based on a Convolutional Neural Network
}

\author{
Guillermo Álvarez-Narciandi \\ Signal Theory and Communications Area \\ University of Oviedo \\ Gijón, Spain \\ alvareznguillermo@uniovi.es
}

\author{
Andrea Motroni \\ Dept. of Information Engineering \\ University of Pisa \\ Pisa, Italy \\ andrea.motroni@ing.unipi.it
}

\author{
Marcos R. Pino \\ Signal Theory and Communications Area \\ University of Oviedo \\ Gijón, Spain \\ mpino@uniovi.es
}

\author{
Alice Buffi \\ Dept. of Energy, Systems, Territory and Constructions Engineering \\ University of Pisa \\ Pisa, Italy \\ alice.buffi@unipi.it
}

\author{
Paolo Nepa \\ Dept. of Information Engineering \\ University of Pisa \\ Pisa, Italy \\ paolo.nepa@unipi.it
}

\begin{abstract}
In this paper, a robust, easy-to-deploy UHF-RFID system to classify transpallet actions at gates or checkpoints has been presented. The system is based on deploying a set of reference RFID tags on the floor of the checkpoint arranged in a matrix form. In addition, a single RFID reader antenna, which is over the checkpoint, is used to query both the reference tags of the system and those that are used identify goods and transpallets. When a transpallet crosses the controlled gate or checkpoint, it directly blocks the reference tags under it. Hence, reference RFID tags under the transpallet are progressively shadowed. As a consequence, if the number of readings of each reference tag is observed versus time, the movement direction of the transpallet can be inferred. This information was used to build images which are then fed to a Convolutional Neural Network (CNN) that classifies transpallet movements in incoming, outgoing or passing through the controlled checkpoint. A total of 159 measurements were acquired for different transpallet trajectories using 24 reference tags and a $\mathrm{CNN}$ was trained showing promising results.
\end{abstract}

Index Terms-RFID, CNN, action classifier

\section{INTRODUCTION}

Radio Frequency IDentification (RFID) technology has contributed to the development of a wide range of applications in retail [1]- [2], healthcare [3]- [4], manufacturing, supply chain and logistics [5]. In particular, in supply chain applications sometimes it could not be necessary to measure the good position in real-time through localization systems [6], but rather to identify specific actions such as entering or exiting

This work has been supported by the Gobierno del Principado de Asturias (PCTI)/FEDER under project IDI/2018/000191; the Ministerio de Educación y Formación Profesional of Spain under the FPU grant FPU15/06431 and EST17/00813, and by the Ministerio de Ciencia, Innovación y Universidades under project ARTEINE (TEC2017-86619-R). This work has also been supported by Region of Tuscany - Regional Government (POR FESR 2014-2020Line 1-Research and Development Strategic Projects) through the Project IREAD4.0 under Grant CUP 7165.24052017.112000028 from a warehouse area. In this regard, different approaches were proposed to implement UHF-RFID gates in the points of interest across the supply chain. Some of them were based on creating controlled reading zones isolated from their surroundings by using tunnel gates [7]. Others rely on extra hardware (e.g. motion sensors or cameras) to determine when a cargo was crossing a point of interest [8]- [9]. Although effective, those solutions require extra space, cost and complexity, which in case of using cameras may also raise privacy concerns. Another solution employed the signature captured from different reader antennas to estimate the movement direction of tagged goods [10]. Other authors proposed the use of low-level parameters extracted from the reader measurements such as the Received Signal Strength Indicator (RSSI) or the timestamps indicating when the data were acquired [11]. Alternatively, the phase of the tag backscattered signal can be employed, by taking advantage from the good movement through a forklift [12] or along a conveyor belt [13].

Recently, machine learning methods were used in the context of RFID systems for localization purposes [14]- [2] and to discriminate tag actions in UHF-RFID gates [15]. This paper presents an innovative approach to classify transpallet movements at checkpoints by using a Convolutional Neural Network (CNN) [16]. CNNs, which have been successfully used for pattern and image recognition among other applications [17], have multiple layers whose purpose is to extract meaningful features from input data. In particular, convolution operations are performed to the input data by means of several filters [16]. The proposed system uses a CNN which employs the data obtained using a single RFID reader antenna and a set of reference tags in order to classify the movement of transpallets. In particular, incoming, outgoing and passing (i.e. moving goods which do not cross the RFID gate) actions are distinguished.

The paper is organized as follows: Section II presents the classification method description and Section III describes the 
results of the experimental analysis. Finally, the conclusion is drawn in Section IV.

\section{METHOD DESCRIPTION}

The proposed system relies on the use of a single RFID reader antenna and several RFID reference tags. The latter are deployed below the antenna and arranged in a matrix form as depicted in Fig. 1. The working principle is based on the fact that when a transpallet or a metallic cart moves over the reference tags, it blocks the line-of-sight between them and the reader antenna. Therefore, the tags which are under the transpallet are shadowed until the transpallet passes through, producing a particular signature that can be used to classify the transpallet action: incoming, outgoing or passing. The concept of shadowing in RFID systems was previously used in [18][19] in device-free localization.

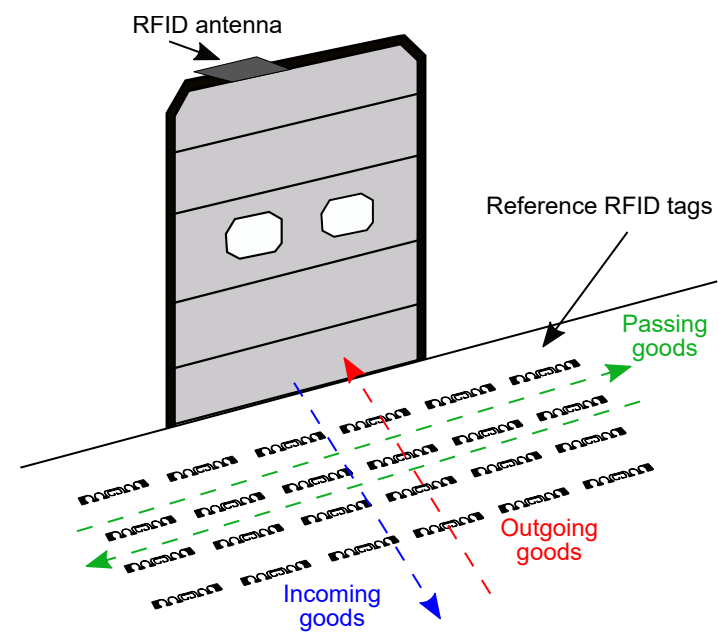

Fig. 1. Basic scheme of the proposed system. Incoming, outgoing and passing movements are shown in red, blue and green arrow, respectively.

The shadowing of reference tags produced by the transpallet movement can cause several effects which are listed below:

- Reduction of collected RSSI samples.

- Significant decrease of tags readings number when the transpallet is moving.

- Missing reads of tags right under the cart.

Specifically, we use the evolution of the number of readings of each reference tag with time to classify transpallet actions (RSSI is more affected by multipath). In particular, as the reader is continuously querying tags, the number of readings of each reference tag is grouped in predefined time slots. The duration of the time slots, denoted as $t_{w i n}$, must be long enough so that all the reference tags can be read within each of them. On the other hand, its duration should not be too long, since in that case the signature produced by the transpallet movement would vanish.

Right after a transpallet moves over the reference tags, the number of readings of each reference tag within the different time slots is arranged in an image form. The obtained image, which summarizes the retrieved information of the transpallet movement over the reference tag, is then used as the input of a CNN in charge of classifying the transpallet action.

The measurement setup used to test the proposed method, which consisted of 24 reference tags arranged in $4 \times 6$ matrix form and a single reader antenna, is depicted in Fig. 2. The space between tags was $30 \mathrm{~cm}$ (in order to reduce the coupling between them) and the width of the metallic cart was $38 \mathrm{~cm}$. It must be pointed out that the separation between tags should be smaller than the width of the cart or transpallet, so it cannot pass between tags without shadowing them. In addition, it should be noted that in a warehouse scenario usually the width of the transpallet or cart carrying goods would be larger than $38 \mathrm{~cm}$, yielding a more visible signature as more tags would be shadowed.

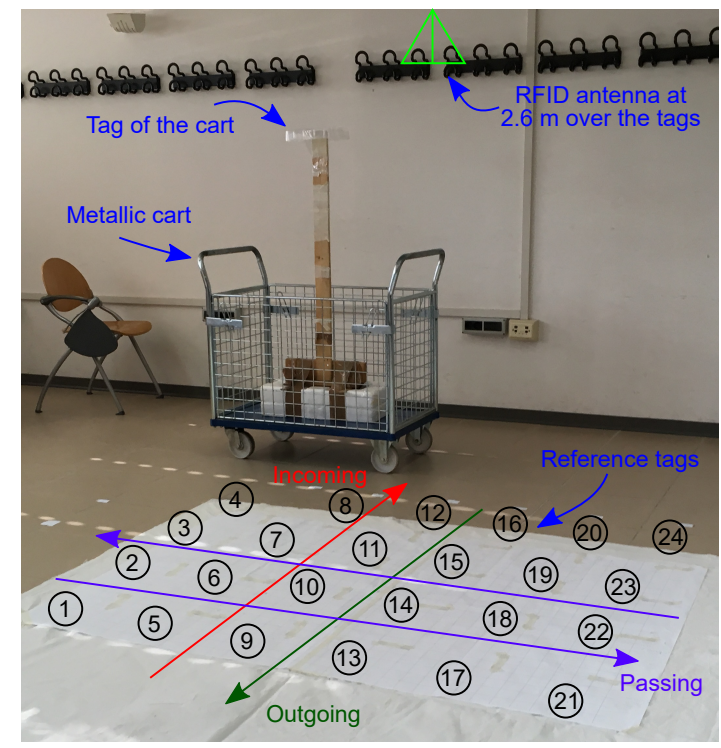

Fig. 2. Measurement setup at the facilities of the University of Pisa. Reference tags numbering is shown to ease the interpretation of the results.

An example of the images which summarize the retrieved information of the transpallet movement for an incoming, an outgoing and a passing trajectory are depicted in Fig. 3a, 3b and $3 c$, respectively. The images were obtained using the setup shown in Fig. 2 by moving a metallic cart. As can be observed, there is a significant reduction of the number of readings of the reference tags when the cart moves over them (dark-blue pixels of the images), which is followed by an increase of the readings as the metallic cart continues its movement outside the check point defined by the grid of reference tags.

An example of these images for an incoming movement, an outgoing trajectory and a passing one are depicted in Fig. $3 \mathrm{a}, 3 \mathrm{~b}$ and $3 \mathrm{c}$, respectively. The images were obtained using a total of 24 reference RFID tags arranged in 4 rows and six columns, as will be described in Section III, moving a metallic cart. As can be observed, there is a significant reduction of the number of readings of the reference tags when the cart moves over them (dark-blue pixels of the images), which is followed by an increase of the readings as the metallic cart continues 
its movement outside the check point defined by the grid of reference tags.

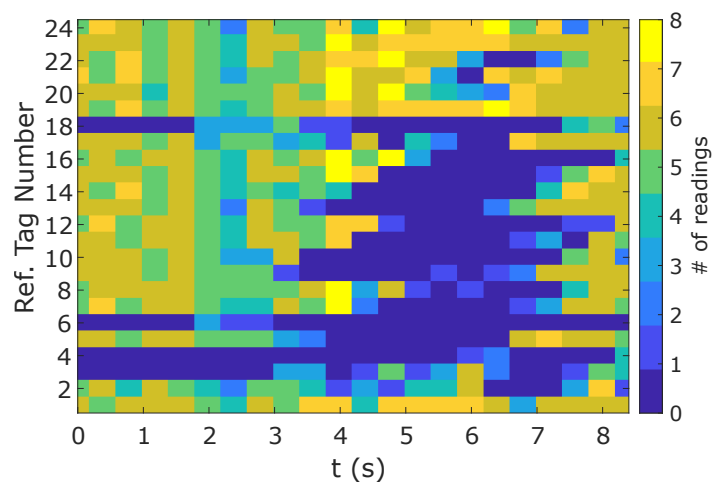

(a)

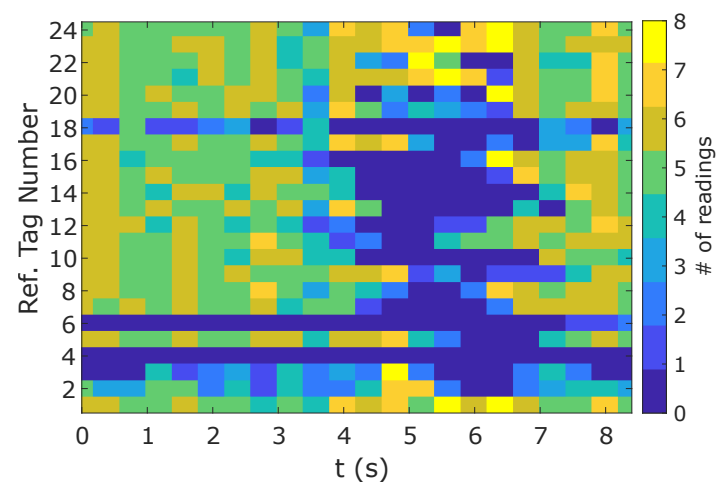

(b)

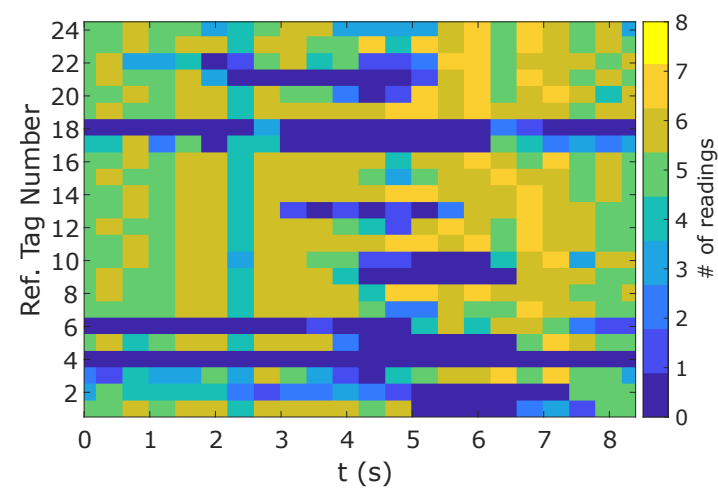

(c)

Fig. 3. Number of readings of each reference tag for the different time slots using $t_{w i n}=400 \mathrm{~ms}$ for an incoming (a), outgoing (b) and passing (c) movement of the metallic cart.

\section{EXPERIMENTAL ANALYSIS}

In order to build a data set to train and test the CNN, 159 measurements were performed. Among those measurements, 50 were incoming movements, 49 were outgoing trajectories and 60 were passing ones. The gathered data were randomly distributed in two groups: the first one (with $60 \%$ of the data) to train the network and the second one (with the remaining $40 \%$ ) to test its performance. The CNN was trained for different sizes of the filters of the convolutional layer and for a different duration of the time slots, $t_{w i n}$. Results are summarized in Table I, where the test accuracy for the different configurations is shown. As can be seen, for $t_{w i n}=400 \mathrm{~ms}$ and a filter of $7 \times 7$ size, a test accuracy of 1 is reached. Therefore, although the size of the data set was modest, the obtained results using the proposed system are promising.

TABLE I. Test accuracy

\begin{tabular}{|c|c|c|c|c|c|c|}
\cline { 2 - 7 } \multicolumn{1}{c|}{} & \multicolumn{7}{c|}{ Size of the filter } \\
\hline$t_{\text {win }}(\mathrm{ms})$ & $4 \times 4$ & $5 \times 5$ & $6 \times 6$ & $7 \times 7$ & $8 \times 8$ & $9 \times 9$ \\
\hline 200 & 0.922 & 0.922 & 0.953 & 0.969 & 0.984 & 0.953 \\
\hline 400 & 0.938 & 0.922 & 0.969 & 1 & 0.953 & 0.984 \\
\hline 600 & 0.922 & 0.953 & 0.891 & 0.938 & 0.906 & 0.906 \\
\hline
\end{tabular}

Finally, once the action of the transpallet is classified, it is necessary to determine which goods were being transported. In order to do so, it is possible to track the phase history of tagged goods and compare it with the one of the tag of the transpallet. With the purpose of validating this approach, during the measurements a set of static tags was deployed to resemble static goods in the surroundings of a check point. In addition, two tags were attached to the metallic cart to simulate a tagged transpallet and the transported goods (see tags on the wood support in Fig. 2). The obtained phase history for the deployed tags for an outgoing trajectory is depicted in Fig. 4. As can be seen, the phase history of static tags is approximately flat whilst the phase history of the tags attached to the cart shows two clear stages. First, the phase grows as the cart moves towards the RFID antenna over the reference tags. Second, it decreases as the cart leaves the antenna behind. As a consequence, static goods can be distinguished from goods on a transpallet crossing a checkpoint by comparing the phase history of the tag of the transpallet with the phase history of other detected tags corresponding to goods.

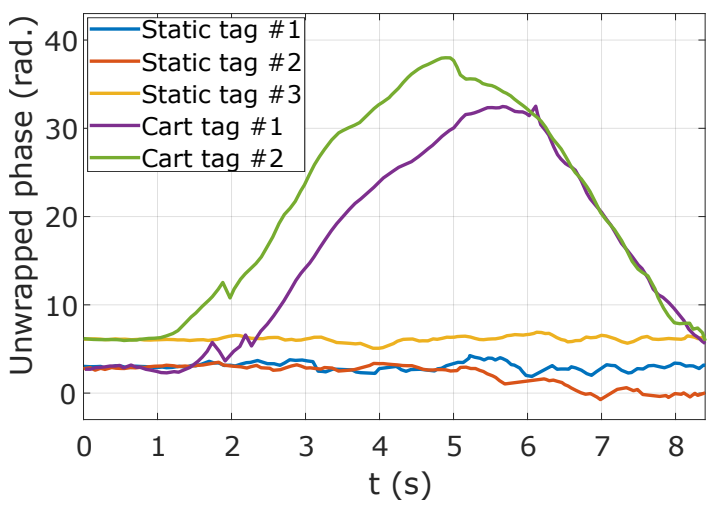

Fig. 4. Phase history of three static RFID tags and two tags attached to the moving metallic cart during an outgoing trajectory.

\section{Conclusion}

In this paper, a novel UHF-RFID system to classify transpallet actions at gates or checkpoints was proposed. The required hardware to deploy the system is minimal. It relies on the use of only one reader antenna and a set of RFID reference tags 
which are deployed on the floor of the controlled checkpoint arranged in a matrix form. Using this setup, when a transpallet moves through a check point, it shadows the tags underneath it. This shadowing effect results in a reduction in number of readings of shadowed tags, yielding a signature of the transpallet movement. In particular, the number of readings of each reference tag (grouped in time slots) was used to create images which are used to feed a CNN, which is able to classify transpallet movements as incoming, outgoing or passing.

The proposed approach was tested deploying a total of 24 reference RFID tags and a metallic cart was used to perform 159 different trajectories (incoming, outgoing and passing). The obtained data was aggregated using different time slots duration and for several parameters of the CNN. Results show a promising performance in terms of validation accuracy. Finally, the phase history was used to discriminate the moving goods from static ones, whose phase history is approximately flat.

\section{REFERENCES}

[1] G. M. Gaukler, "Item-level rfid in a retail supply chain with stock-outbased substitution," IEEE Transactions on Industrial Informatics, vol. 7, no. 2, pp. 362-370, May 2011.

[2] M. Hauser, M. Griebel, and F. Thiesse, "A hidden Markov model for distinguishing between RFID-tagged objects in adjacent areas," in 2017 IEEE International Conference on RFID (RFID), May 2017, pp. 167173.

[3] B. Chowdhury and R. Khosla, "Rfid-based hospital real-time patient management system," in 6th IEEE/ACIS International Conference on Computer and Information Science (ICIS 2007), July 2007, pp. 363 368.

[4] Y. Álvarez López, J. Franssen, G. Álvarez Narciandi, J. Pagnozzi, I. GonzÁlez-Pinto-Arrillaga, and F. Las-Heras, "Rfid technology for management and tracking: e-health applications," Sensors, vol. 18, no. 8, 2018. [Online]. Available: https://www.mdpi.com/1424-8220/18/8/2663

[5] F. Thiesse, E. Fleisch, and M. Dierkes, "Lottrack: Rfid-based process control in the semiconductor industry," IEEE Pervasive Computing, vol. 5, no. 1, pp. 47-53, Jan 2006.

[6] L. M. Ni, Y. Liu, Y. C. Lau, and A. P. Patil, "LANDMARC: indoor location sensing using active RFID," in Proceedings of the First IEEE International Conference on Pervasive Computing and Communications, 2003. (PerCom 2003)., March 2003, pp. 407-415.

[7] R. J. Stine, H. L. Markman, and J. E. Markman, "Shielded portal for multi-reading RFID tags affixed to articles," U.S. Patent 9760826 B1, Mar. 12, 2013.

[8] R. B. Morin, "Method and system for controlling the traffic flow through an RFID directional portal," U.S. Patent 8487 747B2, May 23, 2008.

[9] M. Goller, C. Feichtenhofer, and A. Pinz, "Fusing RFID and computer vision for probabilistic tag localization," in 2014 IEEE International Conference on RFID (IEEE RFID), April 2014, pp. 89-96.

[10] Y. Oikawa, "Simulation evaluation of tag movement direction estimation methods in rfid gate systems," in 2012 IEEE Radio and Wireless Symposium, Jan 2012, pp. 331-334.

[11] T. Keller, F. Thiesse, J. Kungl, and E. Fleisch, "Using low-level reader data to detect false-positive rfid tag reads," in 2010 Internet of Things (IOT), Nov 2010, pp. 1-8.

[12] P. V. Nikitin, G. N. Spiess, H. M. Leland, L. C. Hingst, and J. H. Sherman, "Utilization of motion and spatial identification in mobile RFID interrogator," U.S. Patent 9047 522B1, Nov. 6, 2010.

[13] A. Buffi and P. Nepa, "The sarfid technique for discriminating tagged items moving through a uhf-rfid gate," IEEE Sensors Journal, vol. 17, no. 9, pp. 2863-2870, May 2017.

[14] F. Geigl, C. Moik, S. Hintereggerz, and M. Goller, "Using machine learning and RFID localization for advanced logistic applications," in 2017 IEEE International Conference on RFID (RFID), May 2017, pp. $73-74$
[15] A. Buffi, E. D’Andrea, B. Lazzerini, and P. Nepa, "UHF-RFID smart gate: Tag action classifier by artificial neural networks," in 2017 IEEE International Conference on RFID Technology Application (RFID-TA), Sep. 2017, pp. 45-50.

[16] A. Krizhevsky, I. Sutskever, and G. E. Hinton, "Imagenet classification with deep convolutional neural networks," in Proceedings of the 25th International Conference on Neural Information Processing Systems - Volume 1, ser. NIPS'12. USA: Curran Associates Inc., 2012, pp. 1097-1105. [Online]. Available: http://dl.acm.org/citation.cfm?id=2999134.2999257

[17] K. He, X. Zhang, S. Ren, and J. Sun, "Delving deep into rectifiers: Surpassing human-level performance on imagenet classification," CoRR, vol. abs/1502.01852, 2015. [Online]. Available: http://arxiv.org/abs/1502.01852

[18] B. Wagner, N. Patwari, and D. Timmermann, "Passive RFID tomographic imaging for device-free user localization," in 2012 9th Workshop on Positioning, Navigation and Communication, March 2012, pp. 120125.

[19] E. Eckstein, G. Mazoki, and W. Richie, "Article identification and tracking using electronic shadows created by RFID tags," U.S. Patent 20050012 613A1, Nov. 6, 2010. 